\title{
ASSISTIVE TECHNOLOGY FOR INDEPENDENT LIVING WITH DEMENTIA: STYLIZED FACTS AND RESEARCH GAPS
}

\author{
Michael Bächle ${ }^{\mathrm{a}, \mathrm{n}}$, Stephan Daurer ${ }^{\mathrm{a}}$, Andreas Judt ${ }^{\mathrm{b}}$, Tobias Mettler ${ }^{\mathrm{c}}$ \\ ${ }^{a}$ Baden-Wuerttemberg Cooperative State University Ravensburg Department for Management Information Systems, Marienplatz \\ 2, D-88212 Ravensburg, Germany ${ }^{\mathrm{b}}$ Baden-Wuerttemberg Cooperative State University Ravensburg Department for Informatics, \\ Fallenbrunnen 2, D-88045 Friedrichshafen, Germany \\ 'University of Lausanne Swiss Graduate School of Public Administration, Rue de la Mouline 28, CH-1022 Chavannes-près- \\ Renens, Switzerland
}

Citation: Bächle M, et al. Assistive technology for independent living with dementia: Stylized facts and research gaps. Health Policy and Technology (2018), https://doi.org/10.1016/j.hlpt.2017.12.002

\begin{abstract}
Background: Recent advancement in assistive technologies (AT) have fueled the debate on new, IT-reliant ways of providing cure and care of dementia. Still the impact on practice has been little. With this paper, we want to find out to which extent current studies have discussed the impacts of AT for dementia.

Methods: We conduct a scoping review of the literature on impacts of AT usage in the context of dementia. We search disciplinary (ACM, EMBASE, PsycInfo) as well as cross-disciplinary databases (EBSCO, Web of Science). Based on the identified relevant papers, we extract a list of original statements, which we aggregate to stylized facts. The method of stylized facts is a common research method to derive knowledge in the form of generalized and simplified statements describing interesting characteristics and relationships concerning empirically observable phenomena.

Results: We identify $n=539$ unique articles, out of which $n=36$ report impacts of AT usage in the context of dementia. We aggregate 6 stylized facts that describe common findings. Furthermore, we identify research gaps in this domain. There is little known about the suitable design of social systems around assistive technologies.

Conclusions: While the identified stylized facts indicate how much evidence there is behind certain common statements in the reviewed literature, we additionally find that studies in the area of AT for dementia often neglect the socio-economic and ethical dimension. These are important research gaps for future work.
\end{abstract}

\section{Keywords}

Assistive technology; dementia; independent living; literature review; smart IT; stylized facts 


\section{INTRODUCTION}

For more than a decade, local governments and the European Commission have invested considerable amounts of financial resources into experimentation with assistive technologies (AT) for elderly with the goal to increase autonomy of elderly people so that they can stay longer at home [1]. This has been done based on evidence which showed that elderly people residing in a homecare setting are much more independent and active [2,3] and economically more viable as if they would be treated in long-term care facilities [4]. Accordingly, both technologists and academics have continuously fueled the debate over and made promises that assistive technology (AT) will significantly impact the manner how cure and care will be delivered in near future [3], ultimately resulting in more efficient health systems, and a higher quality of life of millions of senior citizens [5].

However, voices challenging the current (and possibly future) benefits of AT become louder, since largescale social and economic impacts still need to be proven [6]. In this paper, we therefore want to investigate what the current literature tells us about the impacts of AT and how these claims are backed up (if at all) by solid evidence.

To answer the above questions, we conduct a scoped literature review [7]. In what follows, we will briefly explain our understanding and scope of AT in the context of this study. Next, we will describe our literature search process together with the scales and methodology for defining the evidence levels of the identified literature. This is followed by a derivation of stylized facts [8] which were found in the studied literature base and can be used as knowledge in the form of generalized and simplified statements describing interesting characteristics and relationships concerning empirically observable phenomena [8]. We conclude with a critical reflection on research gaps and possible avenues for future research.

\section{BACKGROUND AND SCOPE OF THIS STUDY}

The body of literature regarding AT is due to the fact that many different connotations and meanings coexist. For instance, Marshall [9] defined AT as "[...] any item, piece of equipment, product or system, whether acquired commercially, off-the-shelf, modified or customized, that is used to increase, maintain or improve functional capabilities of individuals with cognitive, physical or communication disabilities." Gibson and colleagues [10] point to the fact that AT does not only refer to electronic equipment, but also quite simple devices such as calendar clocks, as long as they provide assistance with activities of daily 
living or promote activity and enjoyment. In this sense, the concept of AT is used in many fashions for describing automated, IT-reliant, and non-automated, as well as non-customized, semi-customized, and customized assistive appliances and care support services, such as for example designed and adapted to specific contexts (e.g. assisted living [11], homecare [12]) or different diseases (e.g. autism [13], cerebral palsy [14], dementia [15]).

In this study, we focus on IT-reliant solutions for assisting people with mild cognitive impairments, such as early stage dementia patients. We constrain the scope of our literature review, because of three reasons: (i) The literature base about AT is extensive and has rapidly grown in the past years. A limitation with regards to a specific patient group and level of sophistication was needed in order that our analysis yields meaningful and actionable findings; (ii) Dementia affects a large and increasing number of people, not only causing massive social distress, but also great economic losses—around $\$ 236$ billion each year in the U.S. only [16]; (iii) Despite the rapid technological progress manifested by a greater availability of broadband networks, increased miniaturization, and cheaper microchips and sensors, the number of elderly dementia patients using IT-reliant solutions is relatively small [17].

Our study differs from existing studies (e.g. $[17,18])$ in that we seek to characterize the evidence level of the extant body of literature about AT and its impact on dementia patients and caregivers, as opposed to providing an aggregated discussion about the promises and peril of AT in general or a detailed description of specific impacts of a particular tool for dementia patients. The intention is thus to delineate stylized facts-i.e. a set of common findings that can be observed in most studies reporting on IT-reliant assistive tools for dementia patients although applying different methods, focusing on different technologies, or working in different contexts—in order to get a feeling of whether the high hopes and aspirations of policy-makers and technologists are legitimate or not. In the next section, we expand on the literature search process and the methodology regarding how to derive such stylized facts.

\section{METHOD}

Literature reviews are essential for any research [19]. While the assessment of related literature is key to build a foundation of any new research it is also helpful to identify suitable research methods and to derive working hypotheses. Literature reviews often lead to ideas for new studies because they help to 
identify research problems and gaps [20]. There are different approaches to assess the available literature of a certain field or topic [7]. Various guidelines on how to conduct high quality literature reviews are available [21, 22]. There are also some field specific guidelines for health-related reviews [23] or for technology-oriented reviews [24].

For this study, we applied a scoping review [7] with the goal to identify research gaps and to derive stylized facts from the existing literature. In addition to the analysis of previous work, we use stylized facts [8] as a means to generate new knowledge. The method of stylized facts originates from the field of economics and has been successfully used in various other fields of research. The process to derive stylized facts from literature is illustrated in Figure 1 and will be described next.

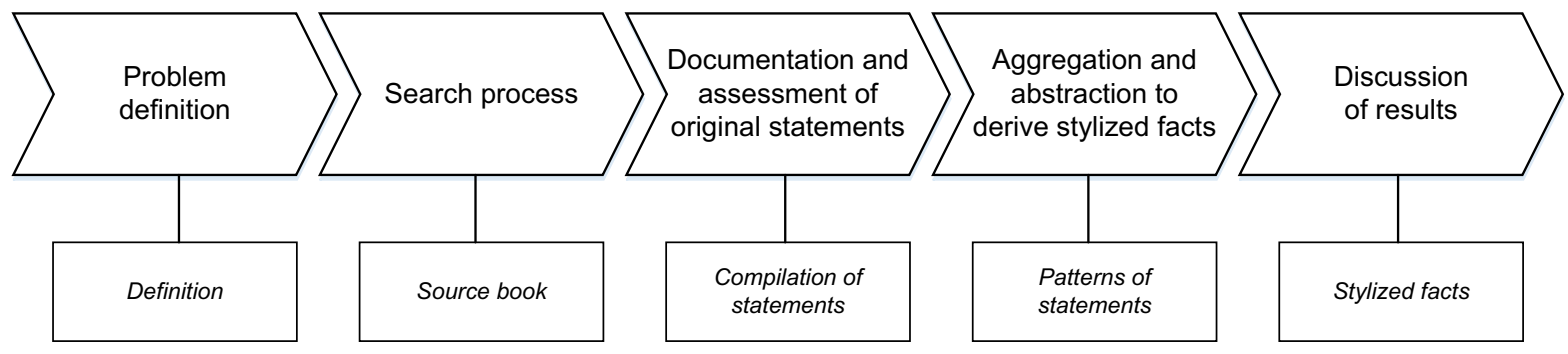

Figure 1. Procedure model for the derivation of stylized facts [8]

Stylized facts constitute knowledge in the form of generalized and simplified statements describing interesting characteristics and relationships concerning empirically observable phenomena [8]. They can be conceptualized as interesting patterns in empirical data documented in different sources. An important characteristic of stylized facts is their focus on the most relevant aspects of observable phenomena by abstracting from details (stylization). Thus, stylized facts are broadly supported and simplified representations of complex relationships that are not necessarily valid in every situation and context. Stylized facts do not aim to represent causal relationships but rather interesting correlations that are observable in reality. In contrast to "classical" hypotheses, stylized facts are statements that have already been confirmed in a certain number of cases, preferably in empirical studies using different methods. Stylized facts can support an inductive development of theory in a bottom up manner.

\section{Problem definition}

Given the demographic changes that developed countries are facing in the next decades, the cure and care of elderly is a significant problem for many public decision makers these days. In fact, providing health to 
a rapidly aging population with fewer resources has become one of the grand challenges of this century for many governments [25]. As described previously, we see dementia as a major challenge for both families and caregivers of affected individuals as well as society as a hole. Albeit there have been massive investments into new technologies, their impact has been modest. In this sense, we would like to systematically explore the extant knowledge base to summarize key findings which hopefully support policy-makers in taking the right conclusions.

\section{Search process}

To identify the relevant literature, we used "Al zheimer" OR "Dementia” OR "mild

cognitive impairment "OR "amnesic" as keywords to characterize our target group together with "information technology" OR "information system" OR "assistance system" OR "assistive system" OR "independent living" to determine the nature of the solution space. We further restricted our search queries to journal articles and conference papers in English language and where full texts were available in disciplinary (ACM, EMBASE, PsycInfo) as well as cross-disciplinary databases (EBSCO, Web of Science). The search queries were performed on the title and abstract fields to ensure that only relevant papers are considered. The queries did not vary between the different databases. As shown in Figure 2, as of July 2016, we obtained a total of 874 articles. 


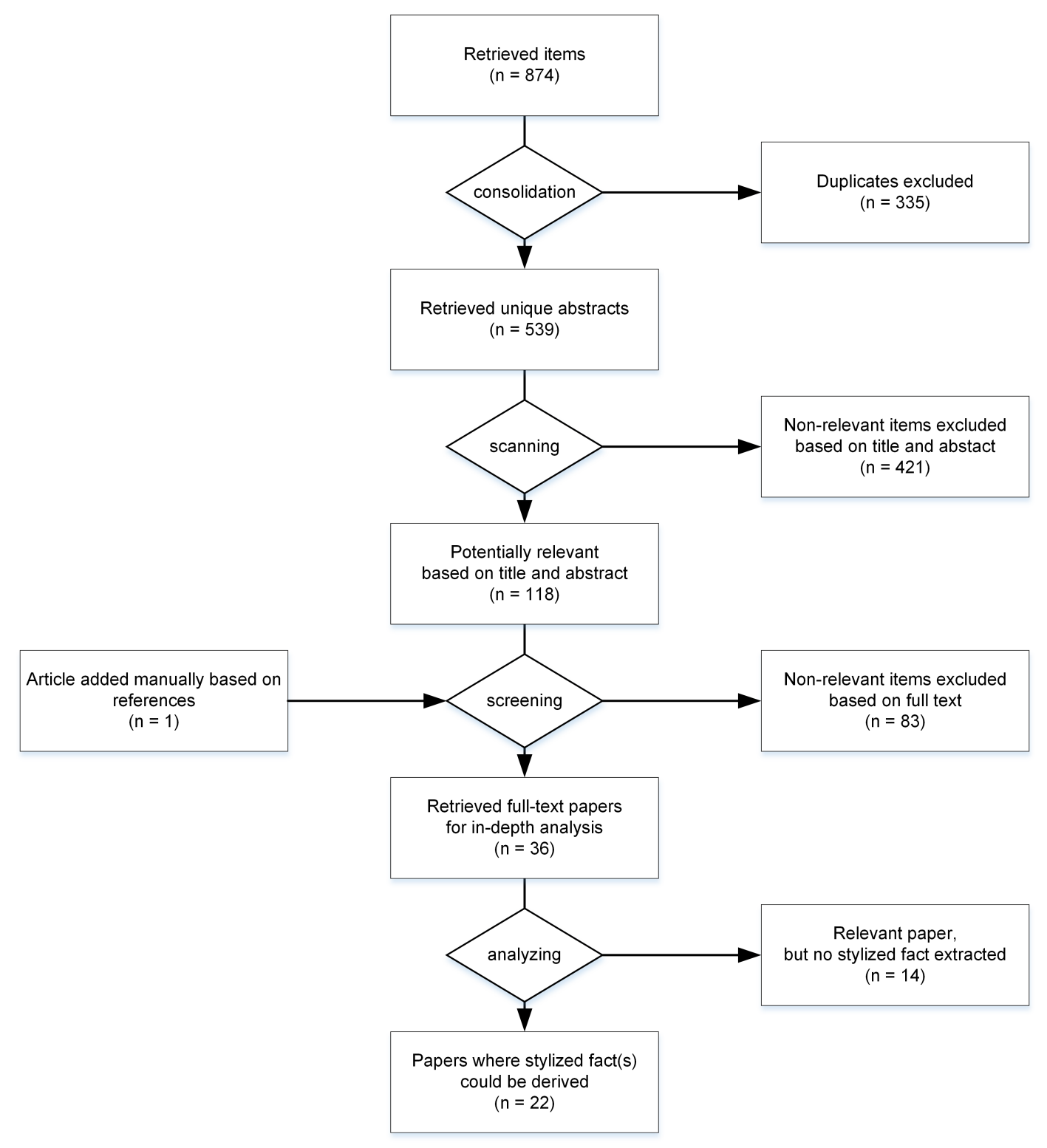

Figure 2. Literature search and assessment process to derive stylized facts

After discarding duplicates (335) and articles with irrelevant content (421) we ended up with a list of 118 potentially relevant articles. Papers were judged to be relevant when their abstracts indicated that the study gave insights into current $\mathrm{AT}$ prototypes or evaluations; in case a judgment regarding the relevance of an article was not possible based on the paper's abstract, we read the introduction, the discussion, and the conclusion section to decide whether to include it or not. This assessment was conducted by two researchers independently. In cases where there was no initial agreement, a third and fourth researcher were involved. One paper was added manually, as it was frequently referenced in the analyzed papers and 
promised novel insights. After carefully reading all papers, we ended up with 36 highly relevant articles, which we used for deriving the stylized facts which we will report later.

\section{Documentation and assessment of original statements}

The first step to obtain stylized facts is to extract a list of unmodified, original statements from the identified literature sources, which are then aggregated and interpreted in a second step. While reading the articles, we extracted 61 original statements (OS) which related to some technological, social, or economic impact of AT on dementia patients and their surroundings (see Appendix A1). According to our research method of stylized facts each statement is further allocated to one of five different evidence levels (EL) which we slightly adapted from Houy et al. [8] for our research field of AT. As described in Table 1, statements with EL 1 are only plausible statements without any empirical evidence. Original statements of EL 2 are narratives or non-generalizable observations like storytelling based case studies or metaphors. EL 3 classifies experiments and other empirical methods which are used in scientifically planned and controlled settings, like a laboratory, but are only applicable under assumptions of very restrictive practical relevance. Statements with EL 4 relate to findings from experiments in clearly defined real-world settings that follow general standard methods and procedures for empirical and experimental studies. They are usually of higher practical relevance in their findings than OS of EL 3 and more generalizable—at least for specific real-world contexts, but still have some restrictive assumptions. Statements with an EL 5 refer to general laws or findings which are applicable to a broad set of real-world settings without restrictive assumptions. 


\begin{tabular}{|c|c|c|}
\hline Evidence Level & Description & Example \\
\hline Level 1 & $\begin{array}{l}\text { Plausible statement that is proven } \\
\text { by mere conceptual consideration } \\
\text { without empirical evidence. }\end{array}$ & "Technique $\mathrm{T}$ is easy to use." \\
\hline Level 2 & Narratives, observations & $\begin{array}{l}\text { "Technique } \mathrm{T} \text { is easy to use. This was illustrated } \\
\text { by three case studies in which T was exemplarily } \\
\text { used." }\end{array}$ \\
\hline Level 3 & $\begin{array}{l}\text { Statement based on empirical or } \\
\text { pseudo-experimental evidence in } \\
\text { an "artificial" context (e.g. lab } \\
\text { experiments) }\end{array}$ & $\begin{array}{l}\text { "Semi-structured interviews with a representative } \\
\text { group showed that technique T is easy to use." }\end{array}$ \\
\hline Level 4 & $\begin{array}{l}\text { Statement based on (randomized) } \\
\text { experiments in a defined "real- } \\
\text { world" context (e.g. field } \\
\text { experiments) }\end{array}$ & $\begin{array}{l}\text { "An experiment with a representative group } \\
\text { showed that the technique } \mathrm{T} \text { is easy to use for a } \\
\text { significantly higher proportion of users }(90 \%) \text {. } \\
\text { Conflicting observations were made for some few } \\
\text { participants." }\end{array}$ \\
\hline Level 5 & $\begin{array}{l}\text { Statement for a clearly specified } \\
\text { context without exception or } \\
\text { which can be deductively derived } \\
\text { from acknowledged statements }\end{array}$ & $\begin{array}{l}\text { "Accepted assumption: Sensors support context- } \\
\text { sensitivity. } \\
\text { Fact: T is a sensor for a specific situation. } \\
\text { Conclusion: T supports context-sensitivity in a } \\
\text { specific AT-relevant situation." }\end{array}$ \\
\hline
\end{tabular}

Table 1. Description of different evidence levels

\section{Aggregation and abstraction to derive stylized facts}

As mentioned previously, the last step is to aggregate and abstract the OS into generalized statements. A generalized statement is the quintessence of several original statements and helps to identify abstract 
stylized facts. Out of the $61 \mathrm{OS}$ and after several rounds of review and discussion, we were able to aggregate 34 generalized statements (see Appendix A2). This intermediary aggregation step builds the foundation for deriving the stylized facts, as it simplifies the interpretation of SF which we will elaborate on in the subsequent section.

\section{DISCUSSION OF RESULTS}

As shown in Table 2, we discovered 6 stylized facts (SF). A SF represents a highly generalized statement, which could be determined across several studies, contexts, and technologies. Each SF will be briefly described next.

\section{Privacy is important but may be overruled by other social values (SF1)}

The first stylized fact (SF1) is based on 13 original statements reported in 5 different references on the matter of privacy of $\mathrm{AT}$ in the context of dementia. In the iterative process to derive this $\mathrm{SF}$, we found only one statement with EL 1, while the rest could be assigned to EL 4. Especially OS42 is very remarkable. In this study of EL 4 the researchers found that dementia patients seem to be rather less concerned with ethical issues with regards to continuous monitoring as media and academia suggests. A possible explanation is that patients in need favor increased quality of life (e.g. safety, mobility, autonomy) over privacy and security of personal data.

\section{Sensors are the centerpiece of AT (SF2)}

For SF 2 we found 5 statements in 4 references, which described sensors as being the primary focus of and means to advance research in the domain of AT. Evidence levels range from 3 to 4 . We believe that this general assumption could be based on experiences from other fields, such as ambient assisted living, where sensors play a dominant role in the design of technological solutions.

\section{Participatory design is essential during the development stage (SF3)}

SF 3 is supported by 14 OS in 11 references, ranging from EL 2 to EL4 (with most studies reporting findings with EL3). SF3 is somewhat an indicator for the need to involve all stakeholders in the early stages of AT design. We think that this finding can be explained by high failure rates of AT projects in the past, where the attention was probably more on the exploration of technological possibilities from a 
medical point of view and less on the needs of patients and their care environment (e.g. family members, caregivers, neighbors).

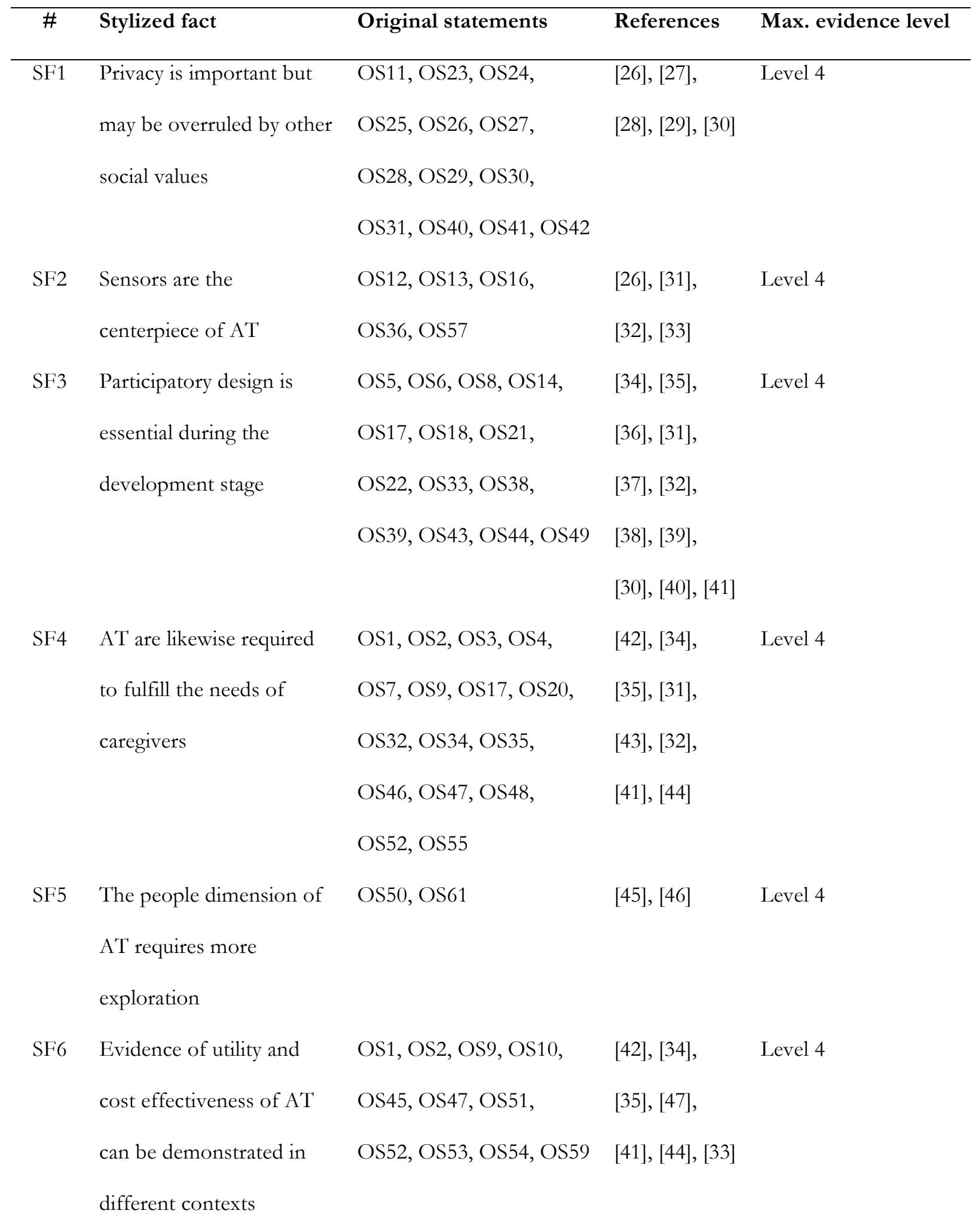

Table 2. Derived stylized facts on assistive technology for people with dementia 


\section{AT significantly support the work of caregivers (SF4)}

We found 16 OS in 8 references with evidence levels between 3 and 4, which stated that AT not only generate positive impacts for patients, but also for their caregivers. In conjunction with SF 3 this is a clear indicator that the successful use of AT is not only a question of sophisticated technological solutions but also a question of identifying the requirements of professional and non-professional caregivers of dementia patients. In this sense, there is a general agreement in the identified literature that AT should be personalized and contextualized to the care environment.

\section{The people dimension of AT requires more exploration (SF 5)}

For SF5 we found 2 references that discussed the role of social aspects regarding the design and use of AT for dementia patients. While one study reported findings with an evidence level of 4 , the other study only had evidence level 1 . Although the literature basis is weak, we came to the conclusion that this is an important finding, worth a stylized fact of its own. We think that the scarce literature base in this area with different evidence levels indicates an urgent research need.

Evidence of utility and cost effectiveness of AT can be demonstrated in different contexts (SF6) This stylized fact (SF6) is based on 11 OS in 7 references, each of which reached evidence level 4. SF6 thus supports the vision that technology can contribute to solving the societal challenge of demographic change and dementia and improving cost effectiveness of health systems. However, because the reported findings did not reach the highest evidence level, it also indicates that this assumption cannot be generalized for all contexts without any limitations or adaptations of proposed solution components. Interestingly, little is reported on the conflicting goals of improving quality of life of patients and cost control. We believe this is a research gap worth exploring in future studies.

When we assess these stylized facts, we find that most of them are centered on technical aspects of AT. We use the framework according to Bostrom/Heinen [49] to show how the stylized facts support the social and the technical subsystem of AT (see Figure 3). The social subsystem comprises of human participants (people with dementia, family members, professional care-givers and health insurance etc.) and the knowledge, skills, attitudes, values and needs they bring to the AT environment. The technical subsystem comprises of devices, tools and techniques needed to transform inputs into outputs in a way 
which enhances the performance of AT for people with dementia. Our stylized facts provide substantial knowledge in the technology and task dimensions of the technical subsystem. However, within the social subsystem there is only some knowledge about the people dimension. No stylized facts could be identified regarding the structure dimension of the social subsystem. We think that this is an important research gap. Further insights within this dimension would help to better understand socio-economic effects of AT in the mid- and long-term.

Social Subsystem

\section{Structure}

No stylized facts found

\section{People}

SF3

Participatory design is essential during the development stage

SF5

The people dimension of AT requires more exploration
Technical Subsystem

\section{Technology}

SF4

AT significantly support the work of caregivers

SF2

Sensors are a centerpiece of AT

Task

SF1

Privacy is important but may be overruled by other social values

SF6

Evidence of utility and cost effectiveness of AT can be demonstrated in different contexts

Figure 3. Stylized Facts in the social and technical subsystems of AT

\section{LIMITATIONS}

The contributions of this study should be viewed in light of the following limitations. Since we base our research on a literature search, other forms of dissemination (e.g. websites, documentation of practitioners, prototypes, etc.) are not considered. Furthermore, only English language papers got included in our study. While we searched many different databases we may not have accessed all relevant databases. In addition, our search terms are somewhat limited and thus we might have missed relevant publications. The development of the different topic categories and the assessment of the evidence level were performed by at least two independent researchers. However, this process cannot eliminate all subjectivity. 
These limitations provide pathways for future research. Further studies might search for approaches on how to set up social subsystems that support the ongoing use of assistive technologies in different language areas or jurisdictions. Different search strategies with different perspectives may add value to the ongoing discussion about best practices and impacts of AT for dementia.

\section{CONCLUSION}

In this paper, we conducted a scoping review for identifying stylized facts regarding the design, use, and appropriation of AT for dementia patients. The systematic analysis of the literature let us conclude that most research puts emphasis on the technical dimension (e.g. requirements elicitation and design) and frequently neglect the ethical aspects of AT (e.g. privacy vs. safety, freedom vs. control). A handful of research projects, which we studied, dealt with questions on ethics and tensions of technology use. We believe this is an important research gap as patients and their care environment would see ethical aspects different in a much-needed state of help. However, this may contradict common privacy policies and cause unforeseen liabilities for health insurers or governmental authorities. Therefore, further research is needed to explore and disentangle underlying ethical tensions to improve our understanding of how to develop viable and acceptable solutions for patients, their caregivers, and governmental authorities.

The scoping review also showed that there is little knowledge about the social subsystem of AT for dementia (see Figure 3). The social subsystem comprises of the human participants (people with dementia, family members, professional care-givers, health insurance etc.) and the knowledge, skills, attitudes, values and needs they bring to the AT environment. The technical subsystem comprises of devices, tools and techniques needed to transform inputs into outputs in a way which enhances the performance of AT for people with dementia. Most of the published research is focused on the technical subsystem. A lot of prototypes and proof-of-concepts in the literature are focused on the technical dimension (i.e. which tasks can be supported with which technology), but nearly nothing is known about the socio-economic effects of AT in the mid- and long-term. In our literature review we have found no AT solutions with evidence level 5 (i.e. statement is valid in specified real-world situations and not only in experiments; see Table 1). This is coherent to our clustering in Fig. 3 with only two stylized facts in the people dimension but none in the structure dimension of the social subsystem of AT. We conclude from this finding that future research should not primarily focus on the development and improvement of technical solutions but 
focus on how to get these technical solutions to become an integrated part of the different socio-

economic systems like private households and senior residences. Research is needed to examine the socioeconomic implications and models to plan, build and run AT solutions in a sustainable manner. Helpful business models are needed for the dissemination and deployment of AT into practice.

\section{ACKNOWLEDGEMENTS}

The authors would like to thank the Internationale Bodensee-Hochschule IBH and the EU for their kind support. This study was supported by the project "IBH Living Lab Active \& Assisted Living" which is part of the Interreg V-Program "Alpenrhein-Bodensee-Hochrhein" which funds are provided by the European Regional Development Fund (ERDF) and the Swiss Confederation. The funders had no role in study design, data collection and analysis, decision to publish, or preparation of the manuscript.

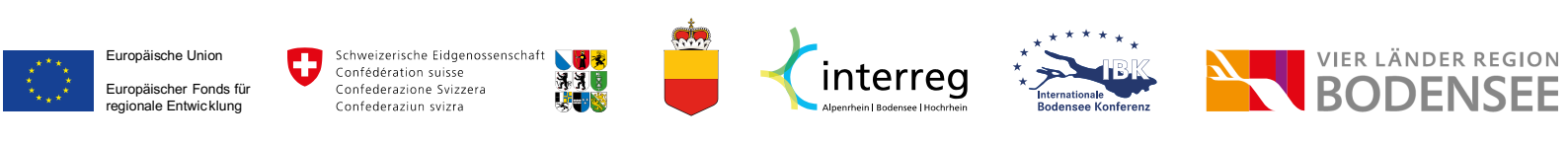

\section{REFERENCES}

1. Kubitschke L, Cullen K, Müller S. ICT \& Ageing: European study on users, markets and technologies - Final report. Brussels: European Commission; 2010.

2. Sun H, De Florio V, Gui N, Blondia C, editors. Promises and challenges of ambient assisted living systems. Proceedings of the 6th International Conference on Information Technology: New Generations; 2009; Las Vegas, USA.

3. Mageroski A, Alsadoon A, Prasad PWC, Pham L, Elchouemi A, editors. Impact of wireless communications technologies on elder people healthcare: Smart home in Australia. Proceedings of the 13th International Joint Conference on Computer Science and Software Engineering; 2016; Khon Kaen, Thailand.

4. Wimo A, Jönsson L, Winblad B. Health economic aspects of dementia. In: Ames D, Burns A, O’Brien J, editors. Dementia. 4 ed. Boca Raton: CRC Press; 2010. p. 327-40.

5. Eccles A, Damodaran L, Olphert W, Hardill I, Gilhooly M. Assistive technologies: Ethical practice, ethical research, and quality of life. In: Sixsmith A, Gutman G, editors. Technologies for Active Aging. New York: Springer; 2013. p. 47-68. 
6. European Commission. Final Evaluation of the Ambient Assisted Living Joint Programme. Brussels: European Commission; 2013.

7. Grant MJ, Booth A. A typology of reviews: an analysis of 14 review types and associated methodologies. Health Information and Libraries Journal. 2009;26(2):91-108.

8. Houy C, Fettke P, Loos P. Stylized Facts as an Instrument for Literature Review and Cumulative Information Systems Research. Communications of the Association for Information Systems. 2015;37(10):225-56.

9. Marshall M. ASTRID: A Social and Technological Response to Meeting the Needs of Individuals with Dementia and their Carers: A Guide to using Technology within Dementia Care. London: Hawker Publications; 2000.

10. Gibson G, Newton L, Pritchard G, Finch T, Brittain K, Robinson L. The provision of assistive technology products and services for people with dementia in the United Kingdom. Dementia. 2014;15(4):681-701.

11. Mileo A, Merico D, Bisiani R. Wireless sensor networks supporting context-aware reasoning in assisted living. Proceedings of the 1st International Conference on Pervasive Technologies related to Assistive Environments; Atherns, Greece2008. p. 54-5.

12. Tudor Car L, El-Khatib M, Perneczky R, Papachristou N, Atun R, Rudan I, et al. Prioritizing problems in and solutions to homecare safety of people with dementia: supporting carers, streamlining care. BMC Geriatrics. 2017;17(1):26.

13. Cassidy S, Stenger B, Van Dongen L, Yanagisawa K, Anderson R, Wan V, et al. Expressive visual text-to-speech as an assistive technology for individuals with autism spectrum conditions. Computer Vision and Image Understanding. 2016;148:193-200.

14. Preston N, Weightman A, Culmer P, Levesley M, Bhakta B, Mon-Williams M. The Cerebral Palsy Kinematic Assessment Tool (CPKAT): feasibility testing of a new portable tool for the objective evaluation of upper limb kinematics in children with cerebral palsy in the non-laboratory setting. Disability and Rehabilitation: Assistive Technology. 2016;11(4):339-44.

15. Altendorf A, Schreiber J. Assistive technology in dementia care: methodological issues in research design. Journal of Assistive Technologies. 2015;9(1):38-47. 
16. Alzheimer's Association. Alzheimer's disease facts and figures 2017 [Available from: http://www.alz.org/facts/

17. Khosravia P, Ghapanchia AH. Investigating the effectiveness of technologies applied to assist seniors: A systematic literature review. International Journal of Medical Informatics. 2016;85(1):1726.

18. Dahler AM, Rasmussen DM, Andersen PT. Meanings and experiences of assistive technologies in everyday lives of older citizens: a meta-interpretive review. Disability and Rehabilitation: Assistive Technology. 2016;11(8):619-29.

19. Webster J, Watson RT. Analyzing the past to prepare for the future: Writing a literature review. MIS Quarterly. 2002;26(2):xiii-xxiii.

20. vom Brocke J, Simons A, Riemer K, Niehaves B, Plattfaut R, Cleven A. Standing on the shoulders of giants: challenges and recommendations of literature search in information systems research. Communications of the Association for Information Systems. 2015;37(9):205-24.

21. Torraco RJ. Writing integrative literature reviews: Guidelines and examples. Human resource development review. 2005;4(3):356-67.

22. Hart C. Doing a literature review: Releasing the social science research imagination: Sage; 1998.

23. Aveyard H. Doing a literature review in health and social care: A practical guide. 3 ed. New York: McGraw-Hill Education; 2014.

24. Boell SK, Cecez-Kecmanovic D. On being 'systematic'in literature reviews in IS. Journal of Information Technology. 2015;30(2):161-73.

25. Joint Institute for Innovation Policy of the European Commission. Investigating in Research and Innovation for Grand Challenges 2012 [Available from: https://ec.europa.eu/research/erab/pdf/erab-study-grand-challanages-2012_en.pdf.

26. Lotfi A, Langensiepen C, Mahmoud SM, Akhlaghinia MJ. Smart homes for the elderly dementia sufferers: identification and prediction of abnormal behaviour. Journal of ambient intelligence and humanized computing. 2012;3(3):205-18.

27. Miskelly F. Electronic tracking of patients with dementia and wandering using mobile phone technology. Age and ageing. 2005;34(5):497-8. 
28. White EB, Montgomery P. Electronic tracking for people with dementia: an exploratory study of the ethical issues experienced by carers in making decisions about usage. Dementia 2014;13(2):216-32.

29. Engström M, Lindqvist R, Ljunggren B, Carlsson M. Relatives' opinions of IT support, perceptions of irritations and life satisfaction in dementia care. Journal of telemedicine and telecare. 2006;12(5):246-50.

30. McCabe L, Innes A. Supporting safe walking for people with dementia: User participation in the development of new technology. Gerontology. 2013;12(1):4-15.

31. Meiland F, Hattink B, Overmars-Marx T, De Boer M, Jedlitschka A, Ebben P, et al. Participation of end users in the design of assistive technology for people with mild to severe cognitive problems; the European Rosetta project. International psychogeriatrics. 2014;26(05):769-79.

32. Evans N, Carey-Smith B, Orpwood R. Using smart technology in an enabling way: a review of using technology to support daily life for a tenant with moderate dementia. British Journal of Occupational Therapy. 2011;74(5):249-53.

33. Khosravi P, Ghapanchi AH. Investigating the effectiveness of technologies applied to assist seniors: A systematic literature review. International Journal of Medical Informatics. 2016;85(1):17-26.

34. Boger J, Mihailidis A. The future of intelligent assistive technologies for cognition: devices under development to support independent living and aging-with-choice. NeuroRehabilitation. 2011;28(3):271-80.

35. Ghorbel M, Betgé-Brezetz Sp, Dupont MP, Kamga GB, Piekarec S, Reerink J, et al. Multimodal notification framework for elderly and professional in a smart nursing home. Journal on multimodal user interfaces. 2013;7(4):281-97.

36. Martin S, Augusto JC, McCullagh P, Carswell W, Zheng H, Wang H, et al. Participatory research to design a novel telehealth system to support the night-time needs of people with dementia: NOCTURNAL. International Journal of Environmental Research and Public Health. 2013;10(12):6764-82.

37. Schikhof Y, Mulder I, Choenni S. Who will watch (over) me? Humane monitoring in dementia care. International Journal of Human-Computer Studies. 2010;68(6):410-22.

38. Lapid MI, Rummans TA, Boeve BF, McCormick JK, Pankratz VS, Cha RH, et al. What is the quality of life in the oldest old? Int Psychogeriatr. 2011;23(6):1003-10. 
39. Malinowsky C, Nygard L, Kottorp A. Using a screening tool to evaluate potential use of e-health services for older people with and without cognitive impairment. Aging \& Mental Health. 2014;18(3):340-5.

40. Mokhtari M, Aloulou H, Tiberghien T, Biswas J, Racoceanu D, Yap P. New trends to support independence in persons with mild dementia: a mini-review. Gerontology. 2012;58(6):554-63.

41. Robinson L, Brittain K, Lindsay S, Jackson D, Olivier P. Keeping In Touch Everyday (KITE) project: developing assistive technologies with people with dementia and their carers to promote independence. International Psychogeriatrics. 2009;21(3):494-502.

42. Armstrong N, Nugent C, Moore G, Finlay D. Using smartphones to address the needs of persons with Alzheimer's disease. Annals of Telecommunications - annales des télécommunications. 2010;65(9):485-95.

43. Engström M, Lindqvist R, Ljunggren B, Carlsson M. Staff members' perceptions of a ICT support package in dementia care during the process of implementation. Journal of Nursing Management. 2009;17(7):781-9.

44. Mao HF, Chang LH, Yao G, Chen WY, Huang WN. Indicators of perceived useful dementia care assistive technology: Caregivers' perspectives. Geriatrics \& Gerontololgy International. 2015;15(8):1049-57.

45. Ballesteros S, Kraft E, Santana S, Tziraki C. Maintaining older brain functionality: A targeted review. Neuroscience Biobehavioral Reviews. 2015;55:453-77.

46. Cahill S, Macijauskiene J, Nygård A-M, Faulkner J-P, Hagen I. Technology in dementia care. Technology and Disability. 2007;19(2, 3):55-60.

47. Pilotto A, D'Onofrio G, Benelli E, Zanesco A, Cabello A, Margeli MC, et al. Information and communication technology systems to improve quality of life and safety of Alzheimer's disease patients: a multicenter international survey. Journal of Alzheimer's Disease. 2011;23(1):131-41.

48. Apostolo J, Holland C, O'Connell MD, Feeney J, Tabares-Seisdedos R, Tadros G, et al. Mild cognitive decline. A position statement of the Cognitive Decline Group of the European Innovation Partnership for Active and Healthy Ageing (EIPAHA). Maturitas. 2016;83:83-93. 
49. Bostrom R, Heinen, JS. MIS Problems and Failures: A Socio-Technical Perspective. Part I: The Causes. MIS Quarterly. 1977;1(4):17-32. 


\section{OS1}

$[42]$

"[Assistive technologies] may be used to relieve carer's burden and help patients remain independent for as long as possible within their own home."

OS2 [34] "IATs [intelligent assistive technologies] have the potential to support ADL [activities of daily living] completion through appropriate compensation of existing cognitive abilities, thus reducing a care recipient's reliance on a caregiver during ADL completion."

OS3 [34] "[...] before an IAT can provide support, it must be able to recognize what $\mathrm{ADL}$ the user is attempting, if he or she requires assistance, and what kind of assistance is appropriate."

OS4 [34] " $[\ldots]$ it must be remembered that the high levels of user dependence and consequences of device failure demand appropriately high levels of IAT reliability and accuracy"

OS5

OS6 [34] “[...] people with cognitive disabilities are considered to be a users, even for people within the same cognitive impairment and demographic, results in an extremely diverse user group." vulnerable population, therefore prototype testing with this group remains a difficult and costly challenge for device developers." of information and the friendliness of the system for the help request." 


\begin{tabular}{|c|c|c|c|}
\hline OS8 & [35] & "Moreover, TV appears as the suitable interaction device for the & Level 3 \\
\hline & & residents. However, a dedicated remote control with bigger & \\
\hline & & button size can certainly be more suitable and then more & \\
\hline & & accessible for elderly people." & \\
\hline OS9 & {$[35]$} & "The healthcare professionals could save time and effectiveness & Level 3 \\
\hline & & when they are directly notified in an abnormal situation or when & \\
\hline & & they know exactly what the assistance specified by the & \\
\hline & & residents." & \\
\hline OS10 & {$[35]$} & "On the professionals side, they demonstrated a high level of & Level 3 \\
\hline & & interest in the interaction with a technical mobile device to & \\
\hline & & manage alerts automatically generated on abnormal situations & \\
\hline & & detection, especially when elderly are dependent and have some & \\
\hline & & cognitive limitations (as some of them tend to use the classical & \\
\hline & & alert button very often, for no particular reason)." & \\
\hline OS11 & {$[26]$} & "Most patients would prefer to use a non-intrusive technology to & Level 1 \\
\hline & & help them with their day-to-day activities." & \\
\hline OS12 & {$[26]$} & "The results presented in this paper show that the start- & Level 3 \\
\hline & & time/duration is the most effective way of representing a large & \\
\hline & & sensor data set. This will also help with the classification of the & \\
\hline & & activities to identify the abnormal behaviour." & \\
\hline OS13 & {$[26]$} & "Echo State Network (ESN) is a very & Level 3 \\
\hline & & promising approach [to predict the future activities] for binary & \\
\hline & & datasets collected from smart environments" & \\
\hline OS14 & {$[36]$} & "Involving the end-users of technology in the development, & Level 3 \\
\hline
\end{tabular}


OS15 [36] " [Small sample sizes and high attrition rates require] the research Level 3 team to demonstrate considerable tenacity and skill to achieve successful deployment."

OS16 [31] "The functionality most often mentioned as relevant and useful Level 3 by persons with dementia was help in cases of emergencies (with movement sensors)."

OS17 [31] "The functionalities most often preferred by carers were support Level 3 with navigation outdoors and the calendar function."

OS18 [31] "The least preferred functionalities were activity support and picture phone-dialling."

OS19 [31] “...] providing an overview of activities that were performed during the day [was] not considered useful”

OS20 [31] "A major concern of the dementia experts was if people with dementia would take the mobile device with them when going outside and if it would be sufficiently charged."

OS21 [31] " [...] in the Rosetta project complementary feedback was Level 3 provided by the different [types of] participants and it was considered useful to also involve persons with dementia, since they provided relevant feedback for the selection and the technical development of the system."

OS22 [37] “[...] an organization may be regarded as a set of people that have a collection of rights, privileges, obligations and responsibilities that are delicately balanced over a period of time through conflict and conflict resolution. Disruption of this balance may give rise to the rejection of the system" 
OS23 [27] "If a person with dementia or wandering issues is wearing a Level 3 phone with GPS capabilities, they can be located with confidence in a high proportion of cases."

OS24 [28] "Protection from harm was described [by carers] as an Level 3 imperative [for electronic tracking], largely taking precedence over protecting the privacy of the person and in some cases, justifying restrictions on autonomy and liberty."

OS25 [28] "Carers frequently referred implicitly and explicitly to freedom Level 3 and independence as important to the quality of life of both the person with dementia and the carer."

OS26 [28] "Safety was predominantly prioritised over privacy." Level 3

OS27 [28] "The relationship between the carer and person with dementia Level 3 appeared to be significant to the decision to use electronic tracking."

OS28 [28] "There was considerable variation in approaches to and the Level 3 value placed on ascertaining the informed consent of the person with dementia before using electronic tracking".

OS29 [28] "The acceptability of covert use appeared to emanate from a Level 3 value system that prioritised safety over privacy and autonomy.”

OS30 [28] "In thinking about privacy, carers appeared to make Level 3 comparisons with alternatives, viewing it [i.e., surveillance technology in dementia care] as less intrusive than constant supervision by a carer."

OS31 [29] "[...] relatives had positive opinions of IT support, and their Level 4 perceptions of practical/logistical irritations decreased after implementation of the IT support package." 
OS32 [43] "The study showed that IT support in dementia care increased Level 4 staff members' satisfaction with their work in several ways."

OS33 [32] “There must be careful assessment of a person's needs and Level 3 matching of the technology to meet these needs."

OS34 [32] "Staff and carers need to have training, commitment and Level 3 support to use the technology."

OS35 [32] "The aim is to develop the process of managing the settings of the flat so that they can be set up by carers and family."

$\operatorname{OS} 36$

[32] "A key challenge is to develop tools to extract complex sensor data so that they can be presented in a clear and easy to understand format, in order to give a clear picture of what is happening in the flat. This will help with the development of reliable and appropriate reports and alerts."

OS37 [38] "Depressive symptoms and cognitive functioning may be Level 4 important predictors of QOL. Interventions designed to address depression and maximize cognitive capabilities may therefore aid in maintaining or improving overall QOL in the oldest old."

OS38 [38] “Clearly, caregivers often perceive the older person's cognitive Level 4 decline, physical impairment and emotional lability as adversely affecting the older person's overall QOL more than the older person does."

OS39 [39] " [...] we cannot assume that people with MCI or AD as groups Level 4 will automatically be non-users of e-health services due to their diagnoses." 
OS40 [30] "GPS devices for people with dementia are considered useful by Level 4 older people, people with dementia and family caregivers to support independence and increase self-confidence."

OS41 [30] "Potential users of the device appear less concerned with the Level 4 ethical issues relating to 'tagging' than those writing in academic journals and the media although they [i.e., potential users] are interested in how the device might be used to promote independence rather than just as a safety precaution offering caregivers peace of mind.”

OS42 [30] "[Potential users] recommended that GPS devices be discreet Level 4 rather than exacerbate any potential stigma."

OS43 [30] "The inclusion of a user-engagement element to the device Level 4 design process provided valuable information about how GPS devices might be used in the real world and specific ideas about different aspects of the device."

OS44 " $[40] \quad[.$.$] we came to the conclusion that in the home environment a$ Level 4 TV set is better suited to provide assistive services than a computer."

OS45 [47] "Relatives/caregivers reported that ICT-systems could be Level 4 significantly more useful for AD patients aged 75-84 than patients aged $<75$ or $\geq 85$ years $(\mathrm{p}<0.0001)$ and with moderate than mild or severe dementia $(p<0.0001)$." and be integrated easily into their daily routines."

OS47 [41] "Suggested areas for functional improvement included two-way communications, flexibility of function as the illness progresses, 
and something to 'guide' them home when out walking or driving."

OS48 [41] "Attention should also be focused on minimizing the size, Level 3 weight and visibility of devices to reduce stigmatization."

OS49 [41] "The study showed that involving people with dementia in the Level 3 process of participatory design is feasible and could lead to devices which are more acceptable and relevant to their needs."

OS50 [45] "In summary, all studies reviewed here, except one, Level 4 demonstrated that social enhancement does contribute to healthier brain aging."

OS51 [44] "These caregivers stated that they would use AT [assistive Level 4 technology] if the technologies were accessible and the cost reasonable, which suggests that these factors are as important as AT research and development."

OS52 [44] "From the experts' perspectives, the indicators of AT [assistive Level 4 technology] devices with HPU [high perceived usefulness] included allows selective accident prevention, has an intuitive interface, is familiar, offers ease of use and simplifies activities."

OS53 [44] "The indicator of AT devices with LPU (lowest perceived Level 4 usefulness) was client prompting."

OS54 [44] "The results suggest that caregivers preferred technologies that Level 4 prevented accidents or emergencies over technologies that informed them of the occurrence of an accident or that only managed the consequences."

OS55 [44] "The results support the importance of using AT devices for Level 4 specific activities with descriptions of specific context." 
OS56 [48] "In sum, non-pharmacological interventions may be associated Level 4 with small cognitive benefits for people with MCI and currently its role can be considered as promising at best."

"Future research should also test whether combination of these strategies (multimodal interventions) is associated with more benefits than monotherapy for MCI patients."

OS57 [33] " [...] remote health monitoring and sensor technologies improve seniors' safety and reduce their risk of falls."

OS58 [33] “...] telemedicine is an effective technology to alleviate seniors' Level 3 health problems."

OS59 [33] "Assistive technologies need to be evaluated not only for their Level 4 feasibility and acceptance, but also for their effectiveness."

OS60 " [33] "...] most of the studies reported the positive effects of Level 4 telemedicine on older adults' health conditions."

OS61 [46] "Development of technology to support activity, entertainment Level 1 and social interaction of people with dementia is so far an almost unexplored field."

Table A1. List of original statements. 


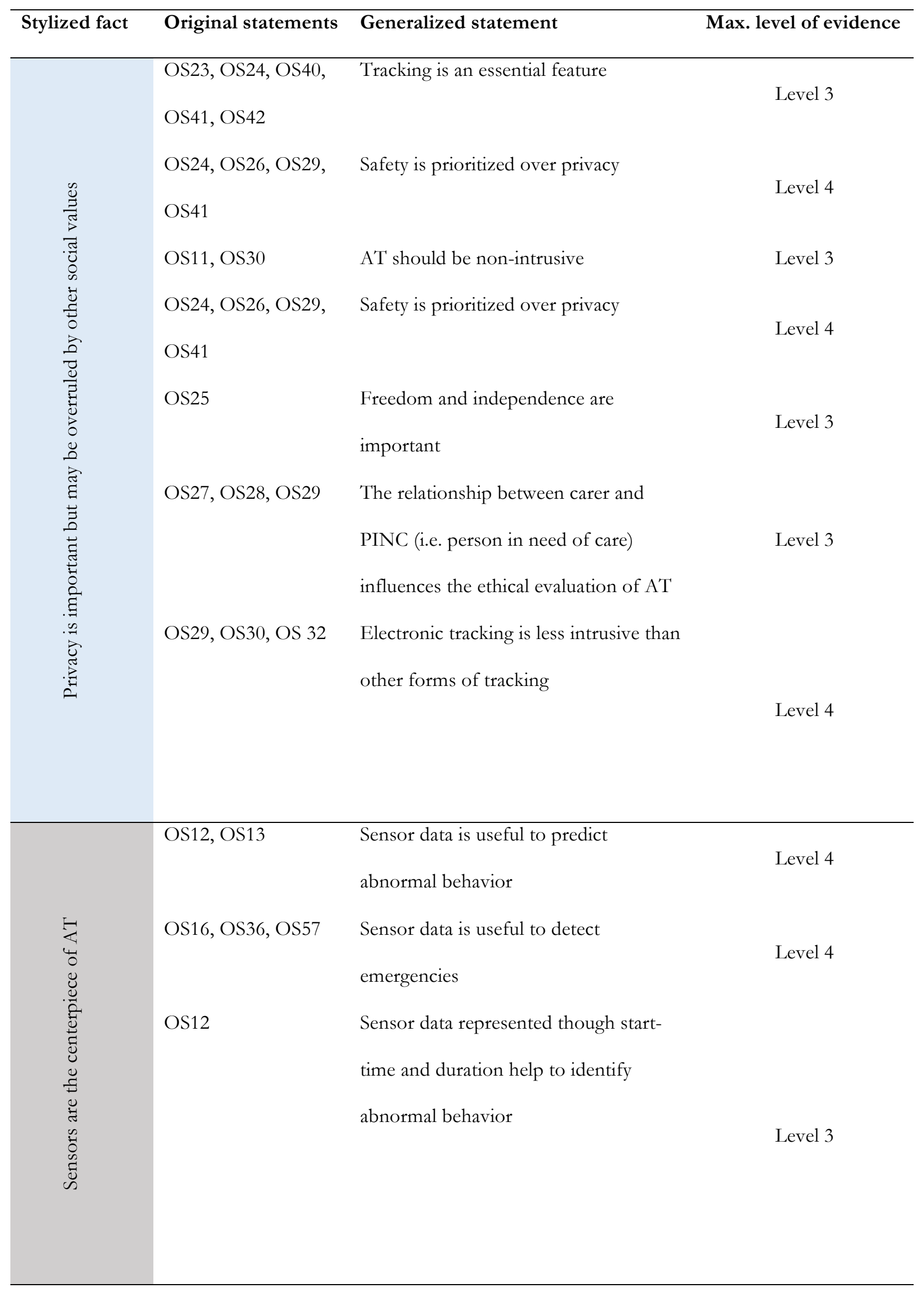




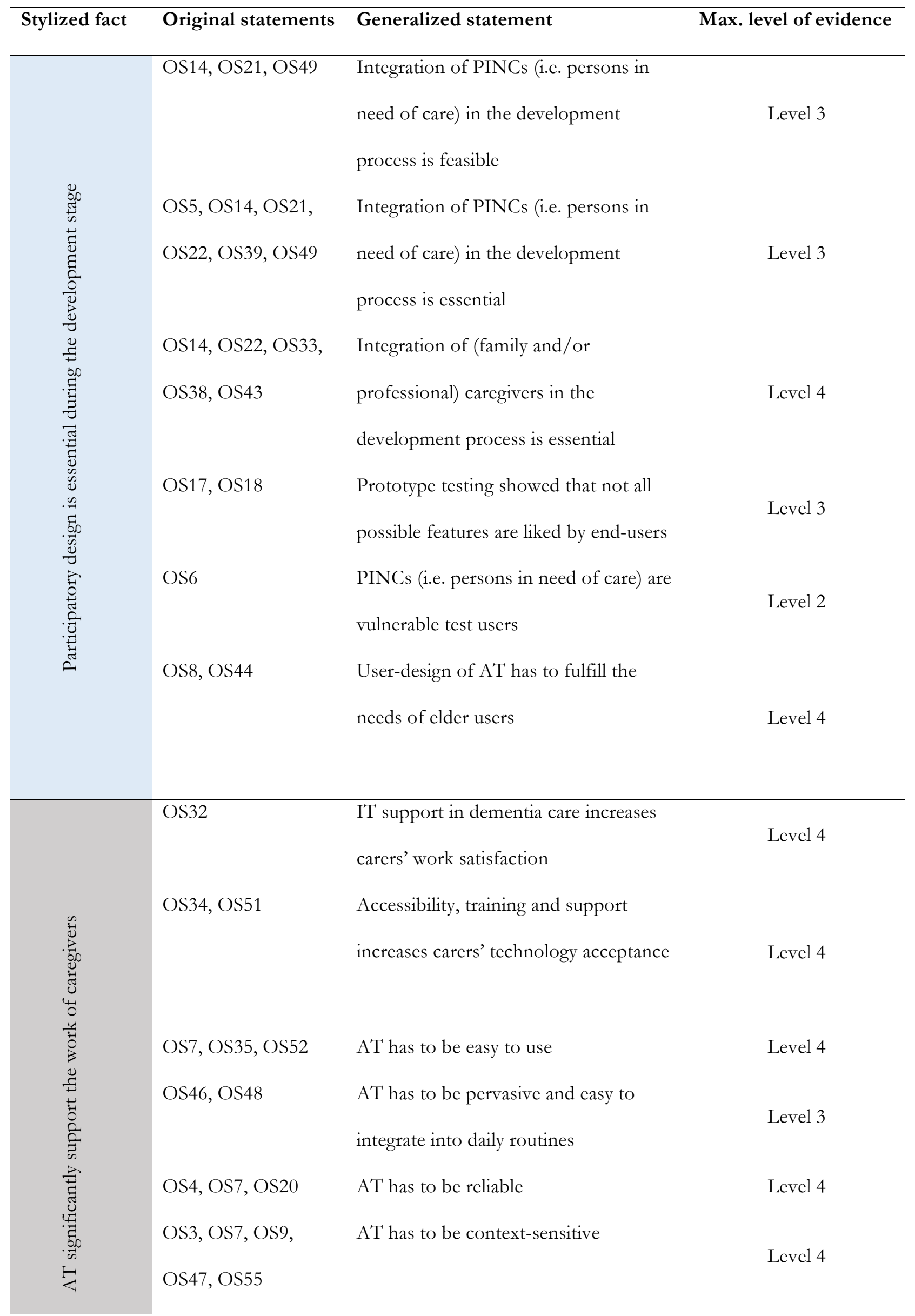




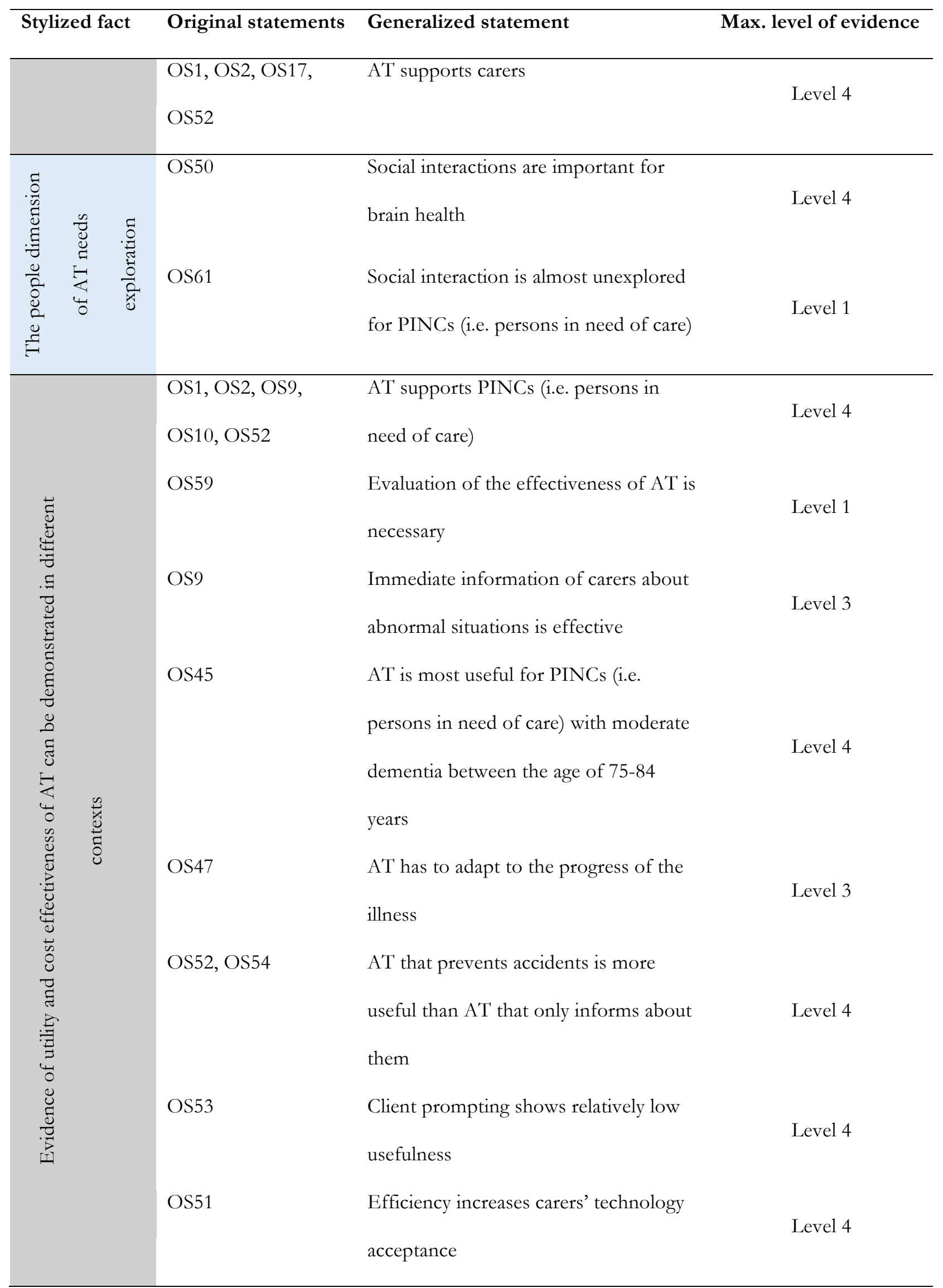

Table A2. List of generalized statements. 